\title{
Drug Discovery Groups
}

National Cancer Institute

\section{Source}

National Cancer Institute. Drug Discovery Groups. NCI Thesaurus. Code C15803.

Self-identified projects in the Biochemistry and Pharmacology Cancer Activity. 